\title{
Ci sono novità in tema di trapianto renale?
}

\author{
Andrea Cavalli, Maria Carmen Luise, Giuseppe Pontoriero \\ S.C. Nefrologia e Dialisi, Ospedale "Alessandro Manzoni", Lecco
}

\begin{abstract}
Any news in kidney transplantation?
A kidney transplant allows better quality of life for patients with end-stage renal disease, even if the number of available organs still falls short of the actual need. Living-donor and HLA-incompatible kidney transplants have been reported to be good options. A systematic literature review has shown that preemptive living donor kidney transplant (when the patient is not yet on dialysis) is associated with better patient and graft survival in addition to lower rates of acute rejection and delayed graft function. A multicenter observational study found that HLAincompatible kidney transplant - although burdened by a high risk of infection and immune complications - can offer patients a survival benefit compared to receiving a kidney from a deceased donor or remaining on the waiting list. In conclusion, preemptive living donor and HLA-incompatible kidney transplants are good therapeutic options in our everyday clinical practice, associated not only with better quality of life but also better survival rates.
\end{abstract}

Keywords: Dialysis, HLA-incompatible transplant, Kidney transplant, Living donor, Preemptive transplantation

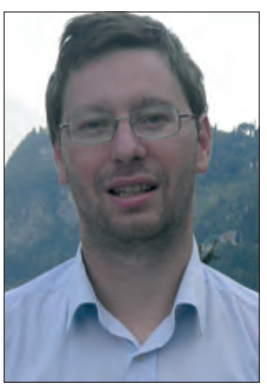

Andrea Cavalli

È ormai noto come il trapianto renale permetta, per coloro che vengono ritenuti idonei, di migliorare la qualità della vita, e probabilmente anche di aumentarne la durata. Tuttavia, a causa della mancanza di organi disponibili, per la maggior parte dei pazienti dializzati occorre aspettare anni, prima di poter ricevere un organo adeguato. È proprio durante questa attesa che le comorbidità associate alla dialisi determinano un progressivo deterioramento dello stato di salute dei pazienti. La possibilità di eseguire un trapianto renale da donatore vivente può quindi espandere il pool di organi disponibili, oltre a rendere possibile di evitare la necessità di dialisi (trapianto pre-emptive), di allestimento di accesso dialitico e, non di meno, determinare un risparmio in termini di costo economico. Alcune osservazioni in merito alla "completa sicurezza" del trapianto pre-emptive sono tuttavia da segnalare (Tab. I).

Accepted: November 1, 2016

Published online: November 16, 2016

Indirizzo per la corrispondenza:

Dr. Giuseppe Pontoriero

S.C. Nefrologia e Dialisi

ASST Lecco, Ospedale "Alessandro Manzoni"

Via dell'Eremo, 9/11

23900 Lecco

g.pontoriero@asst-lecco.it
Recentemente è stata pubblicata una revisione della letteratura e position statement da parte della European Renal Best Practice (ERBP) e del Descartes Working Group (1) in merito ai possibili benefici di un trapianto renale da donatore vivente effettuato prima oppure dopo l'ingresso in dialisi. Sono stati considerati i dati provenienti da 29 studi osservazionali eseguiti dal 1990 in poi, di cui 21 riguardanti trapianti eseguiti in adulti.

Emergevano più frequentemente migliori risultati in termini di sopravvivenza di paziente, trapianto e frequenza di rigetto acuto nel gruppo pre-emptive rispetto a quello dei soggetti in dialisi.

È stata inoltre rilevata una riduzione della sopravvivenza di paziente e trapianto con l'aumento del periodo dialitico, pur evidenziandosi come una permanenza in dialisi inferiore a un anno sembri non avere un impatto significativo sui suddetti outcomes.

In quegli studi che hanno preso in considerazione l'insorgenza di ritardata ripresa funzionale del trapianto, è emersa una netta penalizzazione nei confronti di coloro che erano già in dialisi (2-3.7\% vs 4-9.7\%) (Tab. II).

Va tuttavia sottolineato come gli studi presi in considerazione possano presentare dei rilevanti bias di selezione tali da determinare differenze significative tra i gruppi confrontati e conseguentemente migliori risultati post-trapianto. ̇̀ infatti possibile che i soggetti trapiantati pre-emptive godano di minori comorbidità (minor patologia cardio-vascolare, più elevati livelli di emoglobina e albumina) rispetto a coloro già in dialisi, oltre a differenze di tipo socio-economico (miglior titolo di studio e più frequentemente dotati di assicurazione 
TABELLA I - Possibili vantaggi e svantaggi del trapianto renale eseguito pre-emptive

\begin{tabular}{ll}
\hline Vantaggi & Svantaggi \\
\hline Nessuna necessità di dialisi & $\begin{array}{l}\text { Possibile minor compliance terapeu- } \\
\text { tica legata a non aver sperimentato } \\
\text { lo stato di dializzato (?) }\end{array}$ \\
$\begin{array}{l}\text { Non necessità di allesti- } \\
\text { mento accesso dialitico }\end{array}$ & $\begin{array}{l}\text { Assenza di effetti immunosoppres- } \\
\text { sivi secondari all'uremia (?) }\end{array}$ \\
$\begin{array}{l}\text { Non sviluppo di comorbidità } \\
\text { secondarie allo stato } \\
\text { di dializzato }\end{array}$ & $\begin{array}{l}\text { vivente, a breve termine } \\
\text { Miglior qualità della vita }\end{array}$ \\
Minor costo economico & $\begin{array}{l}\text { Possibili rischi per il donatore } \\
\text { vivente, a lungo termine }\end{array}$ \\
\hline
\end{tabular}

TABELLA II - Vantaggi del trapianto renale da vivente eseguito preemptive vs nel paziente dializzato, in accordo all'articolo di Abramowicz et al (1)

1. Maggior sopravvivenza del paziente

2. Maggior sopravvivenza del rene trapiantato

3. Minor incidenza di rigetto acuto

4. Minor incidenza di ritardata ripresa funzionale del rene trapiantato

privata) e una storia più precoce di accesso alle cure nefrologiche (early referral).

Nonostante questo, il position statement suddetto (1) conclude raccomandando di incoraggiare i programmi di trapianto renale da donatore vivente in modalità pre-emptive, ovviamente informando adeguatamente donatore (e ricevente) circa i potenziali rischi a breve e lungo termine associati a tale decisione. A questo proposito, è stato recentemente pubblicato un articolo inerente lo sviluppo di un algoritmo che permetterebbe di calcolare il rischio di malattia renale cronica terminale nei soggetti donatori (2).

Nel position statement viene inoltre raccomandato che il trapianto renale pre-emptive sia eseguito solo poco prima (qualche mese) dell'ipotetico inizio del trattamento dialitico, in modo tale di evitare l'esposizione troppo precoce di paziente e donatore a inutili rischi. Piuttosto che far riferimento a un prespecificato valore di filtrato glomerulare (al di sotto di $15 \mathrm{~mL}$ / min indicato da alcune Linee guida), viene invece consigliato di considerare il quadro clinico in toto: sintomi, segni e anomalie laboratoristiche difficilmente correggibili.

Sempre rimanendo in tema di trapianto renale da vivente, e ancor più convinti della necessità di sottoporre a trapianto il maggior numero di soggetti idonei alla procedura, è doveroso far riferimento a un'altra recente pubblicazione (3), che ha valutato in uno studio multicentrico osservazionale oltre 1000 soggetti riceventi un rene da donatore vivente HLA incompatibile (HLA-i).
TABELLA III - Tassi di sopravvivenza nei tre gruppi valutati da Orandi et al (3), durante il follow-up

\begin{tabular}{lccc}
\hline & $\begin{array}{c}\text { Trapianto } \\
\text { HLA-i }\end{array}$ & $\begin{array}{c}\text { In lista o trapianto da } \\
\text { donatore deceduto }\end{array}$ & $\begin{array}{c}\text { In lista, ma mai } \\
\text { trapiantato }\end{array}$ \\
\hline 1 anno & $95 \%$ & $94 \%$ & $89.6 \%$ \\
3 anni & $91.7 \%$ & $83.6 \%$ & $72.7 \%$ \\
5 anni & $86 \%$ & $74.4 \%$ & $59.2 \%$ \\
8 anni & $76.5 \%$ & $62.9 \%$ & $43.9 \%$ \\
\hline
\end{tabular}

In particolare, sono stati considerati 1025 adulti sottoposti a trapianto renale HLA-i da donatore vivente in 22 Centri degli Stati Uniti tra il 1997 e il 2011, confrontati con controlli presi dal Registro trapianti, che venivano suddivisi in due categorie: quella di coloro che rimanevano in lista o che ricevevano un trapianto da donatore cadavere e quella di coloro che, pur in lista attiva, non avrebbero mai ricevuto un trapianto. I trapiantati, venivano poi ulteriormente suddivisi in tre gruppi, a seconda del titolo - basso, medio o alto - di anticorpi antidonatore, a cui corrispondevano anzianità dialitiche medie o molto elevate (5.6, 6.9 e 9 anni rispettivamente).

I soggetti che ricevevano un trapianto HLA-i avevano un beneficio statisticamente significativo in termini di sopravvivenza rispetto a entrambi i due gruppi di controllo, a tutte le valutazioni temporali: 1, 3, 5 e 8 anni di follow-up, permettendo un incremento della sopravvivenza - a 8 anni - del $13.6 \%$ e $32.6 \%$ nei confronti dei controlli, rispettivamente trapiantandi da donatore cadavere o che non sarebbero mai stati trapiantati. Il dato entusiasmante è che il vantaggio si manteneva anche andando a suddividere i trapiantati HLA-i in base al grado di iperimmunità: in ogni caso il guadagno in termini di sopravvivenza giustificava il ricorso a una procedura tanto impegnativa come il trapianto da donatore HLA-i (Tab. III). Questi dati, in aggiunta a quelli di uno studio multicentrico risalente ormai al 2011 (4), contribuiscono quindi a fugare i dubbi inerenti il reale beneficio derivante dalle più "spinte" modalità di trapianto eseguite in pazienti ad alto rischio, che tanto sono state ultimamente dibattute in ambito scientifico-trapiantologico.

Per utilizzare un'espressione analoga a quella riportata nell'editoriale (5) di accompagnamento dell'articolo di Orandi, vale la pena quindi correre il rischio di sottoporre un paziente iperimmune a una pesante desensibilizzazione pre-intervento e poi eseguire un trapianto ad alto rischio immunologico (e di possibili complicanze infettive e neoplastiche), perché la sopravvivenza del soggetto sarà comunque superiore a quella di coloro che dovranno aspettare (mediamente per anni e anni) un rene da donatore cadavere o che probabilmente moriranno da dializzati. La recente letteratura presentata, inerente a un affascinante argomento nefrologico come quello del trapianto, declinato in senso pre-emptive e da vivente anche in presenza di pesanti barriere immunologiche, deve continuare a ispirarci nella nostra attività clinica quotidiana. Occorre infatti che il Nefrologo conosca e possa offrire sempre le migliori opzioni 
terapeutiche ai propri pazienti, anche quando queste richiedono grandi sforzi in termini di informazione, tempo, personale coinvolto e costo, oltre che, a volte, rappresentare una sfida a limiti che fino a poco tempo prima si credeva esistessero.

\section{Disclosures}

Financial support: No financial support was received for this submission.

Conflict of interest: The authors have no conflict of interest.

\section{Bibliografia}

1. Abramowicz D, Hazzan H, Maggiore U, et al; Descartes Working Group and the European Renal Best Practice (ERBP) Advisory Board. Does pre-emptive transplantation versus post start of dialysis transplantation with a kidney from a living donor improve outcomes after transplantation? A systematic literature review and position statement by the Descartes Working Group and ERBP. Nephrol Dial Transplant. 2016;31(5): 691-7.

2. Grams ME, Sang Y, Levey AS, et al; Chronic Kidney Disease Prognosis Consortium. Kidney-failure risk projection for the living kidney-donor candidate. N Engl J Med. 2016;374(5): 411-21.

3. Orandi BJ, Luo X, Massie AB, et al. Survival benefit with kidney transplants from HLA-incompatible live donors. N Engl J Med. 2016;374(10):940-50.

4. Montgomery RA, Lonze BE, King KE, et al. Desensitization in HLA-incompatible kidney recipients and survival. N Engl J Med. 2011;365(4):318-26.

5. Rostaing LP, Malvezzi P. HLA-incompatible kidney transplantation - Worth the risk? N Engl J Med. 2016;374(10);982-4. 